\title{
Gasto energético em adolescentes asmáticos com excesso de peso: calorimetria indireta e equações de predição ${ }^{1}$
}

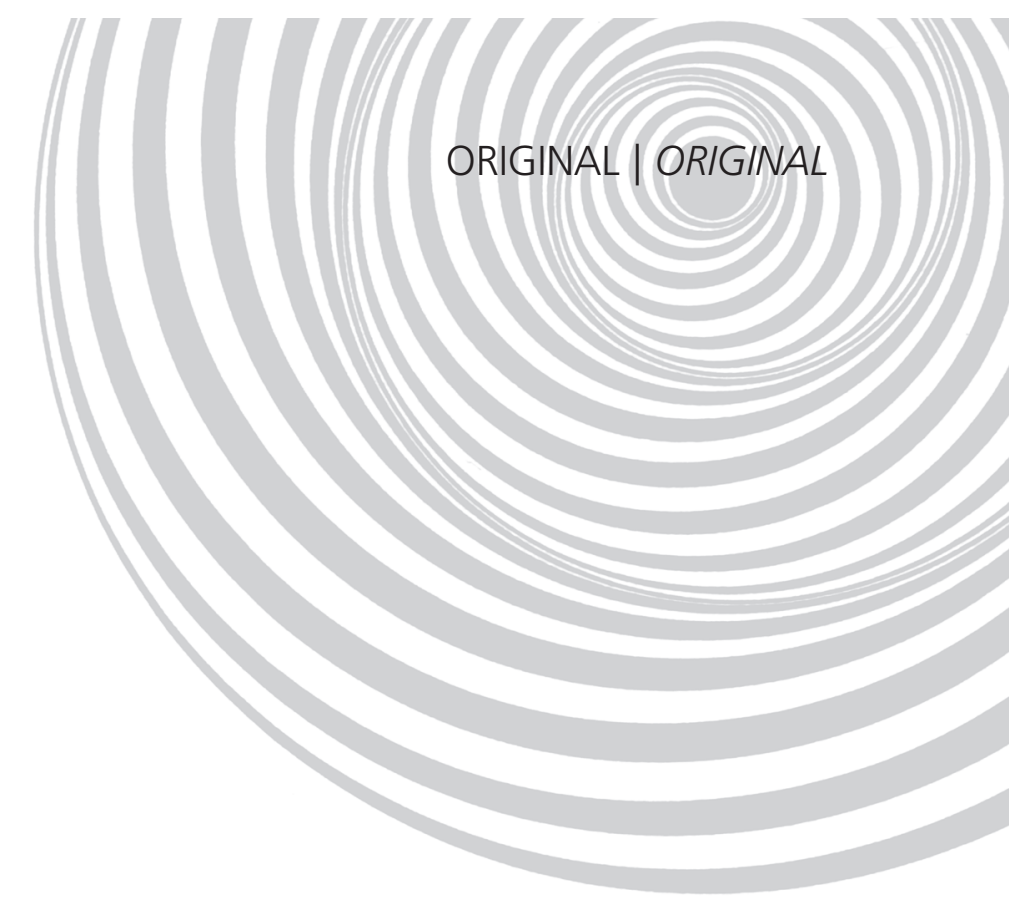

\section{Energy expenditure in overweight, asthmatic adolescents: indirect calorimetry and prediction equations}

Franceliane Jobim BENEDETTI ${ }^{2}$

Vera Lúcia BOSA 2,3

Helena Teresinha MOCELIN ${ }^{4}$

Juliana PALUDO²

Elza Daniel de MELLO2,3

Gilberto Bueno FISCHER ${ }^{2,4}$

\section{RE S U M O}

\section{Objetivo}

Comparar o gasto energético medido por calorimetria indireta com o estimado por equações de predição entre adolescentes asmáticos e não asmáticos.

\section{Métodos}

Trata-se de estudo transversal com 69 adolescentes de 10 a 18 anos. Foram comparados três grupos pareados (asmáticos com excesso de peso, asmáticos eutróficos e não asmáticos com excesso de peso). Para avaliação nutricional utilizaram-se medidas antropométricas e de composição corporal. O gasto energético foi medido por calorimetria indireta e estimado por fórmulas de predição.

$\overline{1}$ Artigo elaborado a partir da dissertação de FJ. BENEDETTI, intitulada "Gasto energético medido por calorimetria indireta em adolescentes asmáticos com excesso de peso". Universidade Federal Rio Grande do Sul; 2008.

2 Universidade Federal do Rio Grande do Sul, Faculdade de Medicina, Programa de Pós-Graduação em Saúde da Criança do Adolescente. R. Ramiro Barcelos, 2400, Santa Cecília, 90035-003, Porto Alegre, RS, Brasil. Correspondência para/Correspondence to: FJ. BENEDETTI. E-mail: <francijb@yahoo.com.br>.

3 Universidade Federal do Rio Grande do Sul, Faculdade de Medicina, Curso de Nutrição, Departamento de Pediatria e Puericultura. Porto Alegre, RS, Brasil.

${ }^{4}$ Hospital da Criança Santo Antônio, Serviço de Pneumologia Pediátrica. Porto Alegre, RS, Brasil. 


\section{Resultados}

Cada grupo foi composto por 23 adolescentes, dos quais 10 do sexo feminino, com média de idade de $M=12,4$, $\mathrm{DP}=2,4$ anos. O gasto energético de repouso, pela calorimetria indireta nos asmáticos com excesso de peso, foi de $M=1550,2$, DP=547,2kcal/dia; nos asmáticos eutróficos, $M=1540,8$, DP=544,2kcal/dia; e nos não asmáticos com excesso de peso, $M=1697,2$, DP=379,8kcal/dia, com resultado semelhante entre os grupos, mesmo quando ajustado pela massa magra e massa gorda $(f=0,186 ; p=0,831)$. Obtiveram-se achados semelhantes entre 0 gasto energético medido pela calorimetria indireta e o estimado pelas fórmulas de predição, com exceção da fórmula de Harris-Benedict, que subestimou o gasto energético nos asmáticos eutróficos e nos não asmáticos com excesso de peso.

\section{Conclusão}

O gasto energético de repouso não foi estatisticamente diferente entre os grupos asmáticos e não asmáticos, mesmo quando ajustado pela massa magra e massa gorda. Para os três grupos, as equações de predição são úteis para estimar o gasto energético de repouso e o gasto energético total.

Termos de indexação: Asma. Calorimetria indireta. Metabolismo energético. Obesidade. Saúde do adolescente.

\section{A B S T R A C T}

\section{Objective}

This study compared the energy expenditure measured by indirect calorimetry with that estimated by prediction equations in asthmatic and non-asthmatic adolescents.

\section{Methods}

This was a cross-sectional study with 69 adolescents aged 10 to 18 years. Three paired groups were compared (overweight, asthmatic adolescents, normal weight, asthmatic adolescents and overweight, non-asthmatic adolescents). Energy expenditure was estimated by indirect calorimetry and prediction equations.

\section{Results}

Each group consisted of 23 adolescents (10 females), with an average age of $M=12.4, S D=2.4$ years. In the group of overweight, asthmatic adolescents, the resting energy expenditure was $M=1550.2, S D=547.2 \mathrm{kcal} /$ day; in the group of normal weight asthmatic adolescents, the resting energy expenditure was $M=1540.8$, $S D=544.2 \mathrm{kcal} / \mathrm{day}$; and in the group of overweight, non-asthmatic adolescents, the resting energy expenditure was $M=1697.2 S D=379.8 \mathrm{kcal} / \mathrm{day}$. The results were similar among groups even when adjusted for lean and fat mass ( $f=0.186 ; p=0.831)$. The total energy expenditure was also similar among groups, except that the Harris-Benedict formula underestimated the energy expenditure in normal weight asthmatic adolescents and overweight, non-asthmatic adolescents.

\section{Conclusion}

The resting energy expenditure was not statistically different among groups of asthmatic and non-asthmatic adolescents, even when adjusted for lean mass and fat mass. For all three groups, the predictive equations were useful for estimating the resting energy expenditure and total energy expenditure.

Indexing terms: Asthma. Indirect calorimetry. Energy metabolism. Obesity. Adolescent health.

\section{N T R O D U ÇÃ O}

A prevalência da asma, assim como a da obesidade, vem aumentando em todo o mundo, a ponto de recentes estudos postularem uma associação entre ambas ${ }^{1}$.

A relação de causalidade entre asma e obesidade não está bem esclarecida, visto que a existência de uma associação significativa não indica necessariamente uma relação de causa-efeito. No entanto, sugere-se que o excesso de peso, entre outros fatores, estaria associado ao aumento da prevalência da asma².

O excesso de peso parece ter efeitos prejudiciais no controle da asma, sendo associado ao aumento dos sintomas respiratórios e da hiper-responsividade brônquica ${ }^{3}$. O aumento do peso tem como um dos fatores determinantes o gasto 
energético, que ainda é pouco estudado em adolescentes asmáticos².

Alguns autores relatam que o principal problema na prática clínica é estimar ou medir as necessidades nutricionais de adolescentes com doenças crônicas. As equações de predição do gasto energético são utilizadas com maior frequência, tanto em contextos clínicos como em condições de vida saudável, pela praticidade e baixo custo. Destaca-se também que, embora existam mais de 190 fórmulas de predição do gasto energético, as mais utilizadas foram desenvolvidas para indivíduos saudáveis. Assim, em pessoas enfermas, essas fórmulas podem contribuir para a sub ou superestimativa do gasto energético, levando a erros na estimativa das necessidades energéticas ${ }^{4}$.

A calorimetria indireta é o padrão-ouro para aferição do Gasto Energético de Repouso (GER). Esse método é fundamental na investigação do metabolismo e na provisão de uma terapia nutricional adequada ${ }^{4}$, embora pouco disponível. Poucos estudos compararam o GER medido por calorimetria indireta com o estimado por fórmulas em adolescentes asmáticos com excesso de peso.

Os estudos mostram que há uma tendência de os indivíduos asmáticos terem o gasto energético aumentado em relação aos não asmáti$\cos ^{5,6}$. Em adolescentes sem doenças associadas, mas com excesso de peso, as evidências, embora contraditórias, sugerem que o gasto energético, quando ajustado para massa magra, está aumentado?.

O objetivo do presente estudo é comparar o gasto energético medido por calorimetria indireta com o estimado por equações de predição entre adolescentes asmáticos e não asmáticos.

\section{MÉ T O D O S}

O delineamento do estudo foi transversal, tendo a coleta de dados ocorrido entre os meses de abril e outubro de 2007.
A população do estudo foi constituída por adolescentes de ambos os sexos, entre 10 e 18 anos, divididos em três grupos: asmáticos com excesso de peso (G1); asmáticos eutróficos (G2); e não asmáticos com excesso de peso (G3). Os pacientes asmáticos estavam em tratamento contínuo com corticoide inalatório há no mínimo um ano e foram acompanhados nos ambulatórios de asma do Hospital Materno Infantil Presidente Vargas e Hospital da Criança Santo Antônio. Os adolescentes não asmáticos foram selecionados numa escola estadual em Porto Alegre, por meio do questionário do estudo International Study of Asthma and Allergies in Childhood (ISAAC), em que negaram apresentar ou ter apresentado sintomas de asma ${ }^{8}$.

Os grupos foram definidos quanto ao estado nutricional de acordo com os percentis de Índice de Massa Corporal (IMC=peso/altura²) propostos por Must et al. ${ }^{9}$, sendo a classificação feita de acordo com os critérios da Organização Mundial da Saúde $(\mathrm{OMS})^{10}$. Como esta não classifica risco nutricional para baixo peso, adotou-se também o proposto por Frisancho ${ }^{11}$. Os grupos foram pareados por idade e sexo, sendo aqueles com excesso de peso (G1 e G3) também pareados pelo percentil de IMC. Foram excluídos adolescentes em uso de lactulose, com IMC $<\mathrm{P} 15$, em hemodiálise ou com comorbidades associadas.

Os adolescentes responderam a um questionário geral e foram submetidos a avaliação antropométrica, recordatório de atividade física e calorimetria indireta.

As medidas antropométricas foram aferidas por nutricionista e estagiária de nutrição previamente treinada, utilizando-se técnicas padronizadas e equipamentos calibrados ${ }^{12}$. Aferiram-se o peso corporal em balança plataforma Plenna ${ }^{\circledR}$ e a estatura, com estadiômetro extensível de parede Sanny ${ }^{\circledR}$.

Avaliaram-se as dobras cutâneas do tríceps e subescapular com compasso Lange ${ }^{\circledR}$, realizando-se três medidas consecutivas, calculando-se a média entre elas ${ }^{13}$. 
Para a avaliação do estágio de maturação sexual realizou-se autoavaliação, considerando-se para os meninos a presença de pelos pubianos e tamanho da genitália e, para as meninas, pelos pubianos e tamanho das mamas, segundo os critérios de Tanner. Classificaram-se em pré-púberes os adolescentes que se encontravam no estágio 1-2 para o sexo masculino e 1 para o feminino; púberes, no estágio 3 para o sexo masculino e 2-3 para o sexo feminino; e pós-púberes, nos estágios 4-5 para ambos os sexos ${ }^{14}$.

A composição corporal foi obtida pelo percentual de gordura corporal calculado pelas equações de Slaughter et al. ${ }^{15}$. A gordura corporal, a massa magra e o percentual de massa magra foram calculados com base no percentual de gordura corporal ${ }^{14}$.

A partir dos registros em prontuário ${ }^{16}$ foram obtidas as características da asma, a dose total de corticoide equivalente à beclometasona e o padrão de gravidade de acordo com o Global Initiative for Asthma (GINA).

O gasto energético em repouso foi medido por calorimetria indireta com analisador de gases TEEM-100 ${ }^{\circledR}$ Inbrasport INC. U.S.A., calibrado de acordo com o fabricante, utilizando-se pneumotacômetro de baixo fluxo (de 4 a 6 litros/20s) e o software Aerograph, que utiliza a equação de Weir para o cálculo do GER ${ }^{17}$.

O indivíduo permanecia em repouso aproximadamente 30 minutos antes da realização da calorimetria e em jejum de três horas ${ }^{4}$. Os asmáticos deveriam suspender os agonistas $\beta 2$-adrenérgicos por 12 horas antecedentes ao exame ${ }^{18}$. A calorimetria indireta foi realizada em ambiente silencioso, com temperatura estável $\left(21\right.$ a $\left.23^{\circ} \mathrm{C}\right)$ e duração de 25 minutos, sendo os cinco primeiros desconsiderados para estabilização dos gases ${ }^{19}$. Para validação do exame foi exigido quociente respiratório entre 0,67 e 1,34. O resultado do GER $(\mathrm{kcal} / \mathrm{min}$ ) foi multiplicado por 1440 para se obter o GER de 24 horas.

Para estimativa do gasto energético utilizaram-se as fórmulas de predição mais utilizadas e recomendadas para adolescentes, apresentadas no Quadro 1.

O Gasto Energético Total (GET) foi determinado multiplicando-se o GER pelo fator atividade, obtido pelo recordatório $24 \mathrm{~h}$ de atividades físicas, conforme valores expressos em Metabolic Equivalents Task - MET ${ }^{20}$. O tempo gasto frente à televisão foi obtido por questionário contendo perguntas referentes à frequência e ao número de horas despendidas na atividade.

Os valores de referência para o cálculo do tamanho da amostra foram obtidos de Tounian et al. ${ }^{21}$, que encontraram o gasto energético de Média $(M)=7060$, Desvio-Padrão (DP) $=1225 \mathrm{kj} / \mathrm{dia}$ no grupo dos indivíduos obesos e $M=5989$, $\mathrm{DP}=888 \mathrm{kj} /$ dia no grupo dos eutróficos. Considerando-se nível de significância de $5 \%$ com poder de $90 \%$, obteve-se um total de 22 indivíduos por grupo ${ }^{21}$. A amostra foi por conveniência consecutiva.

Foram incluídos na pesquisa somente os adolescentes cujos responsáveis aceitaram participar, assinando o termo de consentimento livre e esclarecido aprovado pelos Comitês de Ética em Pesquisa do Hospital Materno Infantil Presidente Vargas (Protocolo 28/06) e do Complexo Hospitalar Santa Casa de Porto Alegre (Protocolo 1444/06).

Quadro 1. Fórmulas de predição para o cálculo do gasto energético.

\begin{tabular}{|c|c|c|}
\hline Fórmulas & Masculino & Feminino \\
\hline Schofield ${ }^{27}$ & $16,25(P)+1,372(A)+515,5$ & $8,365(P)+4,65(A)+200$ \\
\hline $\mathrm{FAO} / \mathrm{OMS}^{28}$ & $17,5 \times P+651$ & $122 \times P+746$ \\
\hline Harris \& Benedict ${ }^{29}$ & $66.47+13.75(P)+5.0(A)-6.76(I)$ & $655.10+9.56(P)+1.85(\mathrm{~A})-4.68(\mathrm{I})$ \\
\hline $10 M^{30}$ & $79-34,2 \times 1+730 \times A+15,3 \times P$ & $22-26 \times I+504 \times A+11,6 \times P$ \\
\hline $\mathrm{RDA}^{31}$ & 10 a 14 anos: $16 \mathrm{kcal} / \mathrm{cm} 15$ a 18 anos: $17 \mathrm{kcal} / \mathrm{cm}$ & 10 a 14 anos: $14 \mathrm{kcal} / \mathrm{cm} 15$ a 18 anos: 13,56kcal $/ \mathrm{cm}$ \\
\hline
\end{tabular}

P: peso em kg; A: altura em cm; I: idade em anos; FAO: Food and Agriculture Organization; OMS: Organização Mundial da Saúde; IOM: Institute of Medicine; RDA: Recommended Dietary Allowance. 
Análise estatística: As variáveis com distribuição simétrica foram descritas através de média e desvio-padrão, e aquelas com distribuição assimétrica, através de mediana e percentis 25 e 75. Para avaliar a distribuição das variáveis, foi aplicado o teste de Kolmogorov-Smirnove, para descrever as variáveis categóricas, foram utilizadas as frequências absolutas e relativas.

Na comparação dos grupos em relação às variáveis contínuas com distribuição simétrica e assimétrica foram utilizadas respectivamente a Análise de Variância (ANOVA) one-way e Kruskal-Wallis. Para complementar a ANOVA (teste post-hoc) foi utilizado o teste de Tukey. Em relação às variáveis categóricas, foi aplicado o teste qui-quadrado de Pearson e, na avaliação das asso- ciações entre as variáveis contínuas, foram empregados os coeficientes de correlação de Pearson. As diferenças foram analisadas com teste $t$ para amostras independentes e pareadas.

O nível de significância adotado foi de 5\%, tendo as análises sido realizadas no programa Statistical Package for the Social Sciences (SPSS) versão 11.5.

\section{RESULTADOS}

Foram arrolados para o estudo 69 adolescentes, compondo três grupos pareados. As características de cada grupo estão apresentadas na Tabela 1. Nota-se que não houve diferenças

Tabela 1. Características demográficas, antropométricas e clínicas dos grupos estudados. Porto Alegre (RS), 2008.

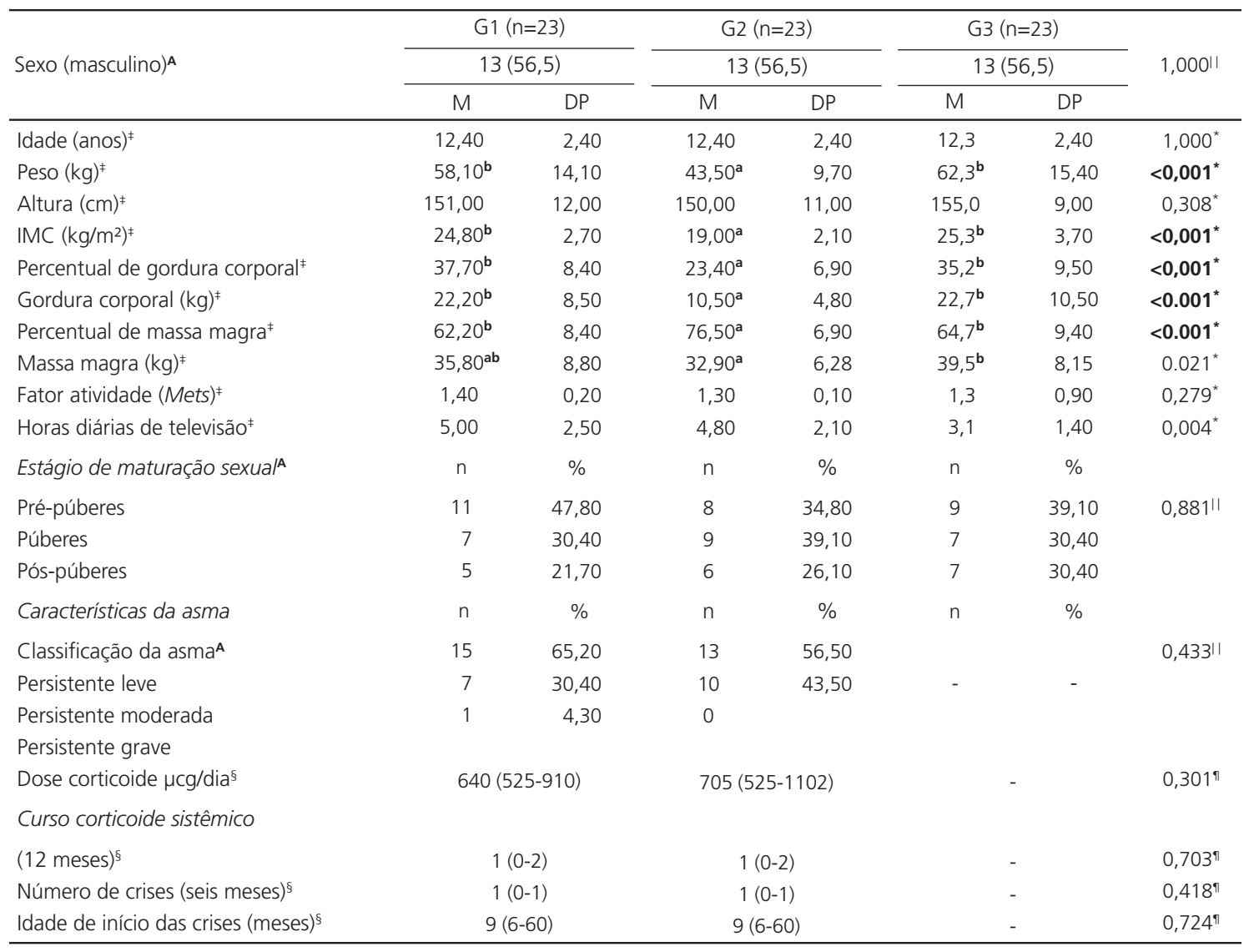

* valor obtido pela Análise de Variância (ANOVA) one-way; 'Valores expressos em número e porcentagem,‡ média (M) desvio-padrão (DP) e ${ }^{\S}$ mediana (P25 - P75) ; " valor obtido pelo teste qui-quadrado de Pearson; " valor obtido pelo teste de Mann-Whitney; ${ }^{a, b}$ letras iguais não diferem pelo teste de Tukey; Numeração em negrito, Valor de $p<0,005$ (valor significamente estatístico).

G1: asmáticos com excesso peso; G2: asmáticos eutróficos; G3: não asmáticos com excesso de peso; IMC: índice de massa corporal; Mets: metabolic equivalents task. 
significativas entre as variáveis sexo, idade e características da asma, mostrando homogeneidade entre os grupos. Nenhum adolescente usava corticoide sistêmico contínuo. Quanto às características antropométricas, observa-se que as variáveis apresentaram diferenças estatisticamente significativas entre os grupos, com exceção da altura, embora esta fosse em média superior entre os não asmáticos. O percentual de gordura corporal foi maior em G1 e G3, porém não houve diferença estatística entre o grupo.

De acordo com a classificação da OMS e de Frisancho, 39\% dos adolescentes com excesso de peso ( $\mathrm{G} 1$ e G3) apresentaram IMC>P95 (obesidade) e $61 \%$ apresentaram IMC entre P85-95 (sobrepeso) $(p<0,001)$.

O GER medido pela calorimetria indireta não foi estatisticamente diferente entre os grupos, mesmo quando ajustado pela massa magra e massa gorda como covariáveis. ( $f=0,186 ; p=0,831)$. Houve associação entre o GER e a massa magra $(f=4,86 ; p=0,031)$, observando-se que quanto maior a massa magra, maior o gasto energético.

A Tabela 2 mostra as comparações do GER medido pela calorimetria indireta, do GET calculado pelo valor medido pela calorimetria indireta multiplicado pelo fator atividade, e do estimado pelas fórmulas de predição, entre os três grupos.
Observa-se que os asmáticos eutróficos apresentavam menor média de gasto energético, com exceção de GER e GET por calorimetria, que não diferiram entre os grupos.

A Tabela 3 apresenta os resultados do gasto energético estimado pelas fórmulas de predição, comparado com o medido pela calorimetria indireta. Obtiveram-se achados semelhantes com os dois métodos de avaliação utilizados, com exceção da fórmula de Harris-Benedict, que subestimou o gasto energético comparado à calorimetria indireta em G2 e G3.

\section{I S C U S S Ã O}

Neste estudo, além do IMC, foram realizadas outras medidas antropométricas para uma avaliação mais precisa do estado nutricional dos participantes. Entre as variáveis antropométricas destaca-se a diferença na média de estatura entre os asmáticos e os não asmáticos, embora sem diferença estatística significativa. Um estudo prévio realizado com a mesma população não encontrou diferença significante na estatura em relação ao uso de corticoide ${ }^{22}$. O alto percentual de gordura corporal apresentado pelos asmáticos pode ser atribuído a fatores associados à asma, entre os quais a baixa frequência de exercícios físicos regulares $^{6}$ e a utilização sistemática de corticoide

Tabela 2. Resultados do gasto energético medido pela calorimetria indireta e estimado pelas fórmulas de predição nos três grupos Porto Alegre (RS), 2008.

\begin{tabular}{|c|c|c|c|c|c|c|c|}
\hline \multirow{2}{*}{ Gasto energético } & \multicolumn{2}{|c|}{ G1 $(n=23)$} & \multicolumn{2}{|c|}{$G 2(n=23)$} & \multicolumn{2}{|c|}{ G3 $(n=23)$} & \multirow{2}{*}{$p^{*}$} \\
\hline & Média & DP & Média & DP & Média & DP & \\
\hline GER calorimetria & 1550,2 & 547,2 & 1540,8 & 544,2 & 1697,2 & 379,8 & 0,492 \\
\hline GET calorimetria & 2088,8 & 688,3 & 2044,6 & 767,2 & 2192,8 & 482,5 & 0,736 \\
\hline IOM & $1553,7^{\mathbf{b}}$ & 239,8 & $1349,9^{a}$ & 167,6 & $1643,6^{b}$ & 267,3 & $<0,001$ \\
\hline Shofield & $1540,7^{\mathbf{b}}$ & 246,6 & $1355,6^{\mathbf{a}}$ & 163,9 & $1604,7^{b}$ & 247,4 & 0,001 \\
\hline FAO/OMS & $1567,4^{b}$ & 236,3 & $1347,9^{\mathrm{a}}$ & 152,7 & $1637,1^{\mathrm{b}}$ & 267,4 & $<0,001$ \\
\hline Harris Benedict & $1484,8^{b}$ & 218,1 & $1310,4^{a}$ & 152,7 & $1559,1^{b}$ & 230,3 & $<0,001$ \\
\hline RDA & 2298,5 & 263,7 & 2283,2 & 259,5 & 2355,9 & 269,7 & 0,618 \\
\hline
\end{tabular}

* valor obtido pela Análise de Variância (ANOVA) one-way; a,b letras iguais não diferem pelo teste de Tukey; Numeração em negrito, Valor de $p<0,005$ (valor significamente estatístico).

G1: asmáticos com excesso peso; G2: asmáticos eutróficos; G3: não asmáticos com excesso de peso; GER: gasto energético de repouso; GET: gasto energético total; IOM: Institute of Medicine; FAO: Food and Agriculture Organization; OMS: Organização Mundial da Saúde; RDA: recommended dietary allowance. 
Tabela 3. Comparação entre o gasto energético medido por calorimetria indireta e estimado pelas fórmulas de predição nos três grupos. Porto Alegre (RS), 2008

\begin{tabular}{|c|c|c|c|c|c|c|}
\hline \multirow{2}{*}{$\begin{array}{l}\text { Variáveis } \\
\text { GER }\end{array}$} & \multicolumn{2}{|c|}{ G1 $(n=23)$} & \multicolumn{2}{|c|}{$\mathrm{G} 2(\mathrm{n}=23)$} & \multicolumn{2}{|c|}{ G3 (n=23) } \\
\hline & $M$ & $\mathrm{DP}$ & $\mathrm{M}$ & $\mathrm{DP}$ & $M$ & DP \\
\hline Calorimetria & 1550,2 & 547,2 & 1540,8 & 544,2 & 1697,2 & 379,8 \\
\hline $\mathrm{IOM}$ & 1553,7 & 239,8 & 1349,9 & 167,6 & 1643,6 & 267,3 \\
\hline Diferença (IC 95\%) & \multicolumn{2}{|c|}{$3,4(-244$ a 252$)$} & \multicolumn{2}{|c|}{$-190,9(-443$ a 61,6$)$} & \multicolumn{2}{|c|}{$-53,6(-185$ a 78,0$)$} \\
\hline \multirow[t]{2}{*}{$p^{*}$} & \multicolumn{2}{|c|}{0,977} & \multicolumn{2}{|c|}{0,131} & \multicolumn{2}{|c|}{0,408} \\
\hline & M & DP & M & DP & M & DP \\
\hline Shofield & 1540,7 & 246,6 & 1355,6 & 163,9 & 1604,7 & 247,4 \\
\hline Diferença (IC 95\%) & \multicolumn{2}{|c|}{$-9,5(-258$ a 239$)$} & \multicolumn{2}{|c|}{$-185(-430$ a 60,2$)$} & \multicolumn{2}{|c|}{$-92,5(-229$ a 44,6$)$} \\
\hline \multirow[t]{2}{*}{$p^{*}$} & \multicolumn{2}{|c|}{0,938} & \multicolumn{2}{|c|}{0,132} & \multicolumn{2}{|c|}{0,176} \\
\hline & M & DP & M & DP & M & DP \\
\hline $\mathrm{FAO} / \mathrm{OMS}$ & 1567,4 & 236,3 & 1347,9 & 152,7 & 1637,1 & 267,4 \\
\hline Diferença (IC 95\%) & \multicolumn{2}{|c|}{$17,2(-229$ a 264$)$} & \multicolumn{2}{|c|}{$-193(-433$ a 47,4$)$} & \multicolumn{2}{|c|}{$-60,1(-189$ a 69,5$)$} \\
\hline \multirow[t]{2}{*}{$p^{*}$} & \multicolumn{2}{|c|}{0,886} & \multicolumn{2}{|c|}{0,110} & \multicolumn{2}{|c|}{0,346} \\
\hline & M & DP & M & DP & M & DP \\
\hline Harris Benedict & 1484,8 & 218,1 & 1310,4 & 152,7 & 1559,1 & 230,3 \\
\hline Diferença (IC 95\%) & \multicolumn{2}{|c|}{$-65,5(-307$ a 176$)$} & \multicolumn{2}{|c|}{$-231(-458$ a $-3,2)$} & \multicolumn{2}{|c|}{$-138(-265 a-10,3)$} \\
\hline$p^{*}$ & \multicolumn{2}{|c|}{0,580} & \multicolumn{2}{|c|}{0,047} & \multicolumn{2}{|c|}{0,035} \\
\hline GET & M & DP & M & $\mathrm{DP}$ & M & DP \\
\hline Calorimetria & 2088,8 & 688,3 & 2044,6 & 767,2 & 2192,8 & 482,5 \\
\hline RDA & 2298,5 & 263,7 & 2283,2 & 259,5 & 2355,9 & 269,7 \\
\hline Diferença (IC 95\%) & \multicolumn{2}{|c|}{$209(521$ a -102$)$} & \multicolumn{2}{|c|}{$238(605 a-128)$} & \multicolumn{2}{|c|}{$163(357,7$ a $-31,3)$} \\
\hline$p^{*}$ & & & & & & \\
\hline
\end{tabular}

*Teste $t$ para amostras pareadas; Numeração em negrito, Valor de $p<0,005$ (valor significamente estatístico).

G1: asmáticos com excesso peso; G2: asmáticos eutróficos; G3: não asmáticos com excesso de peso; GER: gasto energético de repouso; GET: gasto energético total; M média; DP: desvio-padão.

a longo prazo, que é uma rotina no tratamento desses indivíduos 2,3 .

O GER determinado pela calorimetria indireta foi semelhante nos três grupos de adolescentes, mesmo após ajustar para massa magra e massa gorda. Esses achados foram semelhantes a estudos em não asmáticos com excesso de pe$\mathrm{so}^{21}$, mas diferentes do relatado por Van Mil et al. ${ }^{7}$, que identificaram maior Gasto Energético Basal (GEB) nos obesos, mesmo quando ajustada para massa livre de gordura ${ }^{7}$. Por outro lado, estudos com crianças asmáticas sem excesso de peso sugerem que elas têm uma atividade metabólica mais elevada por unidade de massa magra, quando comparadas a não asmáticos ${ }^{5,18}$.

A semelhança no GER entre os grupos pode ser atribuída ao fato de a massa magra, que é o principal preditor do gasto energético, ter sido similar em valores absolutos entre os grupos com excesso de peso $^{23}$. A similaridade entre o GER apresentado em valores absolutos pode dever-se ao fato de esta análise não levar em consideração o peso $(\mathrm{kg})$ dos indivíduos. Cabe ressaltar que os estudos que encontraram maior gasto energético nos asmáticos utilizaram metodologia semelhante, embora tenham sido conduzidos com uma população de crianças menores de onze anos.

Ao comparar os valores das fórmulas de predição entre os grupos, observa-se que o grupo dos asmáticos eutróficos (G2) apresentou menores valores de gasto energético. Isso pode ser justificado, pois as fórmulas de predição requerem dados de peso e estatura, e o gasto energético é proporcional a esses indicadores, que tiveram menores valores nesse grupo.

As comparações das fórmulas de predição com a calorimetria indireta indicaram que os resultados obtidos pelos dois métodos foram similares, com exceção da fórmula Harris-Benedict, que subestimou o gasto energético comparado à 
calorimetria indireta em G2 e G3. Em relação à fórmula de Harris Benedict, achados similares a esse estudo foram encontrados por Fett et al. ${ }^{24}$, cuja fórmula subestimou o GER medido em muIheres $^{23}$. Por outro lado, a maioria dos estudos que avaliaram o gasto utilizando essa mesma fórmula encontraram superestimação do gasto energético ${ }^{7,18,25}$.

Estudos prévios comparando a calorimetria indireta com as fórmulas de predição em não asmáticos encontraram resultados semelhantes. Rodrigues et al. ${ }^{26}$, estudando adolescentes sem excesso de peso encontraram concordância com as equações de Schofield ${ }^{27}$, enquanto Dietz et al. ${ }^{23}$, encontraram concordância com a fórmula FAO/OMS em indivíduos com excesso de peso.

Diferentemente do encontrado neste estudo, Van Mil et al. ${ }^{7}$ verificaram que a FAO/OMS superestimou o GEB em obesos. Schneider \& Mayer ${ }^{25}$ também demonstraram que o emprego de equações de predição, entre elas Shofield ${ }^{27}$, FAO/OMS ${ }^{28}$ e Harris \& Benedic ${ }^{29}$, superestimam os requerimentos energéticos em adolescentes do sexo masculino com excesso de peso.

Observa-se que a literatura não é conclusiva em seus achados quanto ao tema abordado. Divergindo dos estudos que apontam superestimação nas fórmulas de predição, os achados desta pesquisa sugerem que a existência de doença crônica (asma e/ou obesidade) exige maior demanda energética. Nesse sentido, não se observa aumento simultâneo dos resultados quando se comparam os valores medidos pela calorimetria e os estimados pelas fórmulas. Salienta-se também que as fórmulas analisadas foram desenvolvidas para estimar as necessidades de energia de indivíduos saudáveis, com atividades físicas usuais, e elaboradas a partir de indivíduos de origem europeia ou norte-americana $a^{5,7,18}$.

Outro fator a ser considerado é que algumas fórmulas foram construídas com base nos valores do GEB, enquanto neste estudo mediu-se o GER, que se refere ao período pós-absortivo e costuma ser $10 \%$ maior do que o basal. $\mathrm{Na}$ prática clínica essas nomenclaturas se equivalem, já que são poucas as situações em que o indivíduo se encontra em estado de metabolismo basal ${ }^{26}$. Entretanto, tais diferenças devem ser consideradas, principalmente quando se utilizar a fórmula Harris \& Benedict, já que esta apresentou valores entre 10 e $17 \%$ menores do que o medido pela calorimetria indireta.

De acordo com os resultados, as fórmulas de predição Institute of Medicine (IOM), Shofield, FAO/OMS e até mesmo a Harris-Benedict são adequadas para calcular o gasto energético de repouso nessa população, pois a diferença encontrada (DP=200kcal) não apresenta relevância clínica.

Maior atenção deve ser concedida ao gasto energético total, uma vez que a inadequação das equações de predição pode acarretar erros na estimativa da necessidade energética, principalmente nos adolescentes com excesso de peso.

O GET, obtido pela multiplicação do GER pelo fator atividade, pode superestimar o gasto energético, resultando em um balanço energético positivo, o que seria inaceitável para esses grupos. O GET obtido calculado pela Recommended Dietary Allowance (RDA) foi semelhante ao obtido pelo GER medido pela calorimetria mais o fator atividade. Também não mostrou diferenças entre os grupos, talvez por não levar em consideração o peso.

Em relação ao GET, salienta-se a importância da obtenção correta do fator atividade, uma vez que se observaram valores semelhantes entre os grupos, embora se tenha realizado apenas um recordatório de atividades físicas. Neste estudo, o tempo despendido com televisão foi maior no grupo dos asmáticos, corroborando o achado de Chen et al., que indicaram ser comum nesses pacientes a pouca prática de exercícios ${ }^{6}$.

Este estudo apresenta algumas limitações metodológicas, entre as quais a aferição precisa do fator atividade, que poderia interferir no GET. Salienta-se que na presente pesquisa há prevalência de pacientes com asma leve ou moderada, não podendo os resultados ser extrapolados para 
pacientes com asma grave. Deve-se considerar que nestes últimos o gasto energético possa ser maior que o estimado pelas equações de predição.

A partir dos resultados do presente estudo, conclui-se que o gasto energético de repouso foi semelhante entre os grupos estudados e que as equações de predição IOM, Shofield, FAO/OMS e Harris-Benedict são adequadas para essa população, inclusive a RDA para o cálculo do gasto energético total.

\section{A GRADECIMENTOS}

Ao Conselho Nacional de Desenvolvimento Científico e Tecnológico, à Escola Estadual de Educação General Flores da Cunha, à ONG de Peito Aberto, à Imbrasport e a Vânia Naomi Hirakata pelas análises.

\section{COLABORADORES}

F.J. BENEDETTI concebeu estudo e participou da redação do artigo. G.B. FISCHER e E.D. MELLO participaram da orientação e revisão do artigo. H.T. MOCELIN, V.L. BOSA e J. PALUDO participaram da definição do projeto e da redação do artigo.

\section{REFERÊ N CIAS}

1. Beuther AD, Sutherland ER. Overweight, obesity, and incident asthma: a meta-analysis of prospective epidemiologic studies. Am J Respir Crit Care Med. 2007; 175(7):661-6.

2. Ford $\mathrm{E}$. The epidemiology of obesity and asthma. J Allergy Clin Immunol. 2005; 115(5):897-909.

3. Camilo DF, Ribeiro JD, Toro ADC, Baracat ECE, Barros Filho AAB. Obesidade e asma: associação ou coincidência? J Pediatr (Rio de Janeiro). 2010; 86(1):6-14.

4. Matarese LE. Indirect calorimetry: technical aspects. J Am Diet Assoc. 1997; 97 (2):154-60.

5. Zeitlin SR, Bond S, Wootton S, Gregson RK, Radford $M$. Increased resting energy expenditure in childhood asthma: does this contribute towards growth failure? Arch Dis Child. 1992; 67(11): 1366-69.

6. Chen $Y$, Dales R, Krewski D. Leisure-time energy expenditure in asthmatics and non-asthmatics. Respir Med. 2001; 95(1):13-8.
7. Van Mil EG, Westerterp KR, Kester AD, Saris WH. Energy metabolism in relation to body composition and gender in adolescents. Arch Dis Child. 2001; 85(1):73-8.

8. Solé D, Vanna AT, Yamada E, Rizzo MC, Naspitz CK. International study of asthma and allergies in childhood (ISAAC) written questionnaire: validation of the asthma component among Brazilian children. J Investig Allergol Clin Immunol. 1998; 8(6):376-82.

9. Must A, Dallal GE, Dietz WH. Reference data for obesity: $85^{\text {th }}$ and $95^{\text {th }}$ percentiles of body mass index (wt/ht2) and triceps skinfold thickness. Am J Clin Nutr. 1991; 53(4):839.

10. World Health Organization. Development of a WHO growth reference for school-aged children and adolescents. Bull World Health Organ. 2007; 85:660-7.

11. Frisancho AR. Anthropometric standards for the assessment of growth and nutritional status. Ann Arbor: The University of Michigan Press; 1990. p.48-53.

12. Lohman TG, Roche AF, Martorrel R. Anthropometric standardization reference manual. Books, Champagne(IL): Books Human Kinetic; 1988.

13. Frisancho AR. New norms of upper limb fat and muscle areas for assessment of nutritional status. Am J Clin Nutr. 1981; 34(11):2540-45.

14. Tanner JM. Growth at adolescence. $2^{\text {nd }}$ ed. Oxford: Blackwell Scientific Publications; 1962.

15. Slaughter MH, Lohman TG, Boileau RA, Horswill CA, Stillman RJ, Bemben DA. Skinfold equations for estimation of body fatness in children and youth. Hum Biol. 1988; 60(5):709-23.

16. Global Initiative for Asthma. The Global Strategy for Asthma Management and Prevention (updated 2007); the updated Pocket Guides and the complete list of references examined by the Committee are available on the GINA website. [cited 2008 Feb 20]. Available from: <www. ginasthma.org>.

17. Weir JB. New methods for calculating metabolic rate with special reference to protein metabolism. J Physiol. 1949; 109(1-2):1-9.

18. Maffeis C, Chiocca E, Zaffanello M, Golinelli M, Pinelli L, Boner AL. Energy intake and energy expenditure in prepubertal males with asthma. Eur Respir J. 1998; 12(1):123-9.

19. Hoher JH, Teixeira PJZ, Hertz FT, Moreira JS. A comparison between ventilation modes: how does activity level affect energy expenditure estimates? J Parenter Enteral Nutr. 2008; 32(2):176-83.

20. Farinatti PTV. A Brazilian-Portuguese version of the compendium of physical activities: a contribution 
to exercise physiology researchers and professionals. Rev Bras Fisiol Exerc. 2003; 2(2): 117-208.

21. Tounian PNC, Dumas N, Veinberg F, Girardet JP. Resting energy expenditure and substrate utilization rate in children with constitutional leanness or obesity. Clin Nutr. 2003; 22(4):353-57.

22. Arend EE, Fischer GB, Debiasi M, Schmid H. Inhaled corticosteroid treatment and growth of asthmatic children seen at outpatient clinics. J Pediatr. 2006; 82(3):197-203.

23. Dietz WH. Critical periods for the development of obesity. Am J Clin Nutr. 1994; 59(5):955-9.

24. Fett C, Fett WCR, Marchini JS. Gasto energético de repouso medido vs. estimado e relação com a composição corporal de mulheres. Arq Bras Endocrinol Metab. 2006; 50(6):1050-8.

25. Schneider P, Meyer F. As equações de predição da taxa metabólica basal são apropriadas para adolescentes com sobrepeso e obesidade? Rev Bras Med Esporte. 2005; 11(3):93-6.

26. Rodrigues G, Moreno LA, Sarría A, Fleta J, Bueno $M$. Resting energy expenditure in children and adolescents: agreement between calorimetry and prediction equations. Clin Nutr. 2000; 21(3):255-60.

27. Schofield WN. Predicting basal metabolic rate, new standards and review of previous work. Hum Nutr Clin Nutr. 1985; 39c(1):5-41.

28. World Health Organization. Energy and protein requirements. Report of a joint FAO/WHO/UNU expert consultation. Geneva: World Health Organization; 1985. (WHO Technical Report Series 724).

29. Harris JA, Benedict FG. A Biometric study of basal metabolism in man. Boston: Carnegie Institution of Washington; 1919.

30. Institute of Medicine of the National Academies. Dietary reference intakes for energy. Washington (DC): National Academy Press; 2002.

31. National Research Council. Recommended dietary allowances. Washington (DC): National Academy Press; 1989.

Recebido em: 22/10/2008

Versão final reapresentada em: 13/10/2010 Aprovado em: 3/11/2010 